\title{
Area-level global and local clustering of human Salmonella Enteritidis infection rates in the city of Toronto, Canada, 2007-2009
}

Csaba Varga ${ }^{1,2^{*}}$, David L. Pearl ${ }^{1}$, Scott A. McEwen ${ }^{1}$, Jan M. Sargeant ${ }^{1,3}$, Frank Pollari ${ }^{4}$ and Michele T. Guerin ${ }^{1}$

\begin{abstract}
Background: Salmonella enterica serotype Enteritidis (S. Enteritidis) remains a major foodborne pathogen in North America yet studies examining the spatial epidemiology of salmonellosis in urban environments are lacking. Our ecological study combined a number of spatial statistical methods with a geographic information system to assess area-level heterogeneity of $\mathrm{S}$. Enteritidis infection rates in the city of Toronto.

Methods: Data on S. Enteritidis infections between January 1, 2007 and December 31, 2009 were obtained from Ontario's surveillance system, and were grouped and analyzed at the forward sortation area (FSA)-level (an area signified by the first three characters of the postal code). Incidence rates were directly standardized using the FSA-level age- and sex-based standard population. A spatial empirical Bayes method was used to smooth the standardized incidence rates (SIRs). Global clustering of FSAs with high or low non-smoothed SIRs was evaluated using the Getis-Ord G method. Local clustering of FSAs with high, low, or dissimilar non-smoothed SIRs was assessed using the Getis-Ord Gi* and the Local Moran's I methods.
\end{abstract}

Results: Spatial heterogeneity of $S$. Enteritidis infection rates was detected across the city of Toronto. The non-smoothed FSA-level SIRs ranged from 0 to 16.9 infections per 100,000 person-years (mean =6.6), whereas the smoothed SIRs ranged from 2.9 to 11.1 (mean =6.3). The global Getis-Ord G method showed significant $(p \leq 0.05)$ maximum spatial clustering of FSAs with high SIRs at $3.3 \mathrm{~km}$. The local Getis-Ord Gi* method identified eight FSAs with significantly high SIRs and one FSA with a significantly low SIR. The Local Moran's I method detected five FSAs with significantly high-high SIRs, one FSA with a significantly low-low SIR, and four significant outlier FSAs (one high-low, and three low-high).

Conclusions: Salmonella Enteritidis infection rates clustered globally at a small distance band, suggesting clustering of high SIRs in small distinct areas. This finding was supported by the local cluster analyses, where distinct FSAs with high SIRs, mainly in downtown Toronto, were detected. These areas should be evaluated by future studies to identify risk factors of disease in order to implement targeted prevention and control programs. We demonstrated the usefulness of combining several spatial statistical techniques with a geographic information system to detect geographical areas of interest for further study, and to evaluate spatial processes that influenced S. Enteritidis infection rates. Our study methodology could be applied to other foodborne disease surveillance data.

\footnotetext{
*Correspondence: cvarga@uoguelph.ca

'Department of Population Medicine, Ontario Veterinary College, University

of Guelph, Guelph, ON N1G 2W1, Canada

${ }^{2}$ Ontario Ministry of Agriculture, Food and Rural Affairs, Guelph, ON N1G 4Y2,

Canada

Full list of author information is available at the end of the article
} reproduction in any medium, provided you give appropriate credit to the original author(s) and the source, provide a link to the Creative Commons license, and indicate if changes were made. The Creative Commons Public Domain Dedication waiver (http://creativecommons.org/publicdomain/zero/1.0/) applies to the data made available in this article, unless otherwise stated. 


\section{Background}

Salmonellosis continuously poses a significant health burden to human populations globally, affecting annually an estimated 93.8 million persons worldwide [1]. In Canada, an estimated 109,384 non-typhoidal Salmonella infections are acquired domestically, of which $80 \%$ are considered to be foodborne [2]. Within the last decade, an increase in the number of Salmonella enterica serotype Enteritidis ( $S$. Enteritidis) infections has been reported in Canada [3], the United States of America [4], and the European Union [5], such that $S$. Enteritidis has become the top serotype among the non-typhoidal salmonellae. Salmonella Enteritidis infections in humans have typically been associated with consumption of contaminated chicken products $[6,7]$ and eggs $[8,9]$. However, salmonellosis has recently been linked to other factors, including international travel $[10,11]$, demographic $[12,13]$ and socioeconomic $[14,15]$ characteristics, and animal contact $[7,16]$.

Country- or region-level studies have used various spatial epidemiological methods to identify clustering of health conditions, including notifiable gastrointestinal illness [17], giardiasis [18], campylobacteriosis [19, 20], influenza B [21], Escherichia coli O157 [22, 23], dengue fever $[24,25]$, traumatic brain injury [26], stroke [27], and myocardial infarction [27]. Moreover, city-level studies have evaluated spatial differences in neighbourhood-level infection rates of rotavirus in Berlin, Germany [28], pandemic influenza A in Hong Kong [29], tuberculosis in Linyi City, China [30], and typhoid fever [31-33] and dengue [34] in the Dhaka metropolitan area of Bangladesh.

Our study area involved the city of Toronto-the capital of Ontario, Canada located on the shore of Lake Ontario in the southern part of the province (Fig. 1). In 2009, an estimated 2.7 million people lived in the city, accounting for $21 \%$ of Ontario's total population [35]. Toronto's forward sortation areas (FSAs; areas signified by the first three characters of the postal code; see Study design and data sources section) have diverse age- and sex-based populations that can affect arealevel infection rates, due to sex differences of salmonellosis rates [36, 37], and younger and older residents' higher salmonellosis rates [13, 16, 38]. Standardization of area-level infection rates based on the age and sex distribution of the population has been recommended to overcome this problem [39]. Moreover, infection rates in small population areas can become unstable and unreliable. The spatial empirical Bayes (SEB) smoothing method has been proposed to reduce the random variation of infection rates linked with these areas $[39,40]$.

Despite the abundance of research studies that have assessed large scale (country- or region-level) spatial processes that influence foodborne infections, few studies have assessed small scale (city- or FSA-level) spatial clustering of salmonellosis rates. Small area studies in urban environments are useful as a first step for identifying areas with high infection rates, where future studies can be conducted to identify novel individual-level risk factors, which can assist in the design of local prevention and control programs $[21,28]$. Our retrospective, population-based, ecological study used a systematic approach that combined spatial exploratory and statistical methods with a geographic information system (GIS) (Fig. 2), to evaluate the spatial heterogeneity of $S$. Enteritidis infection rates across the city of Toronto. Moreover, two local spatial cluster detection methods were compared to identify their strengths and weaknesses in analyzing small-scale infectious disease data.

\section{Methods}

\section{Study design and data sources}

Forward sortation areas are well-delimited areas signified by the first three characters of the postal code; they are established by the Canada Post Corporation based on the mail distribution zones of postal facilities. Forward sortation area-level population estimates and FSA cartographical boundary files were acquired from the 2006 Census of Canada [35, 41].

In Ontario, salmonellosis is a reportable disease under provincial legislation [42]. A diagnosis of salmonellosis is made after isolation of Salmonella spp. (excluding Salmonella Typhi or Paratyphi) from an appropriate clinical sample (the majority are stool samples) by public health, hospital, or private laboratory staff [43]. All isolates are sent to the Toronto Public Health Laboratories for confirmation and serotyping using the KauffmannWhite scheme [44]. Salmonellosis cases must be followed up by local public health unit staff, and investigation findings must be reported to the Ontario Ministry of Health and Long-Term Care (MOHLTC) through the integrated Public Health Information System (iPHIS). This surveillance system is a repository for all reportable disease data in Ontario; no major modifications in salmonellosis reporting requirements, or testing or case follow-up protocols were noted during the study period, which makes salmonellosis case ascertainments robust and reliable.

We obtained case information from all reported $S$. Enteritidis infections from the city of Toronto between January 1, 2007 and December 31, 2009 that were captured within iPHIS.

\section{Statistical analysis}

Spatial heterogeneity of $S$. Enteritidis infection rates was assessed by following several analytical steps, which are outlined in Fig. 2, and described in detail below. 


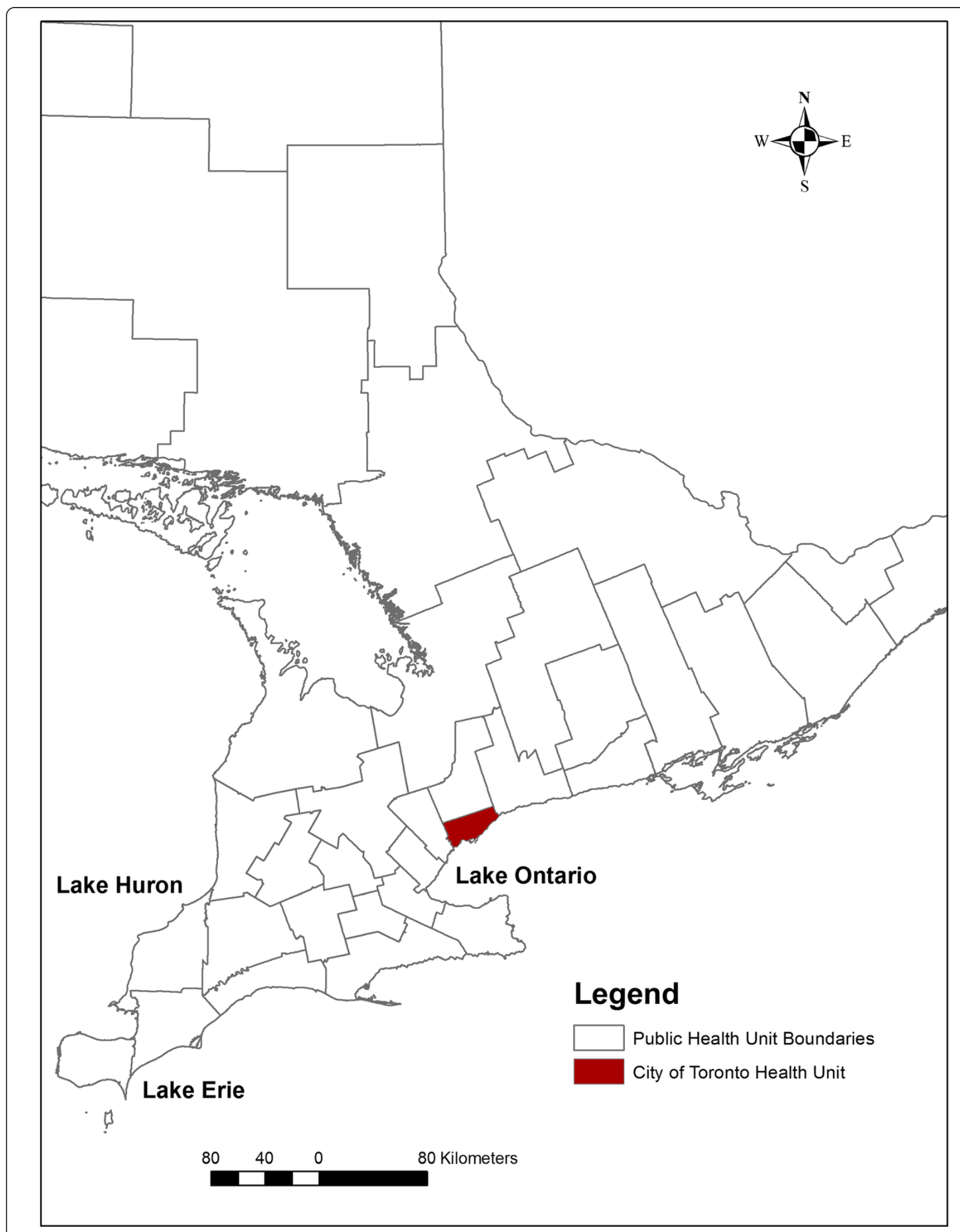

Fig. 1 Map of Ontario, Canada highlighting the location of the study area 


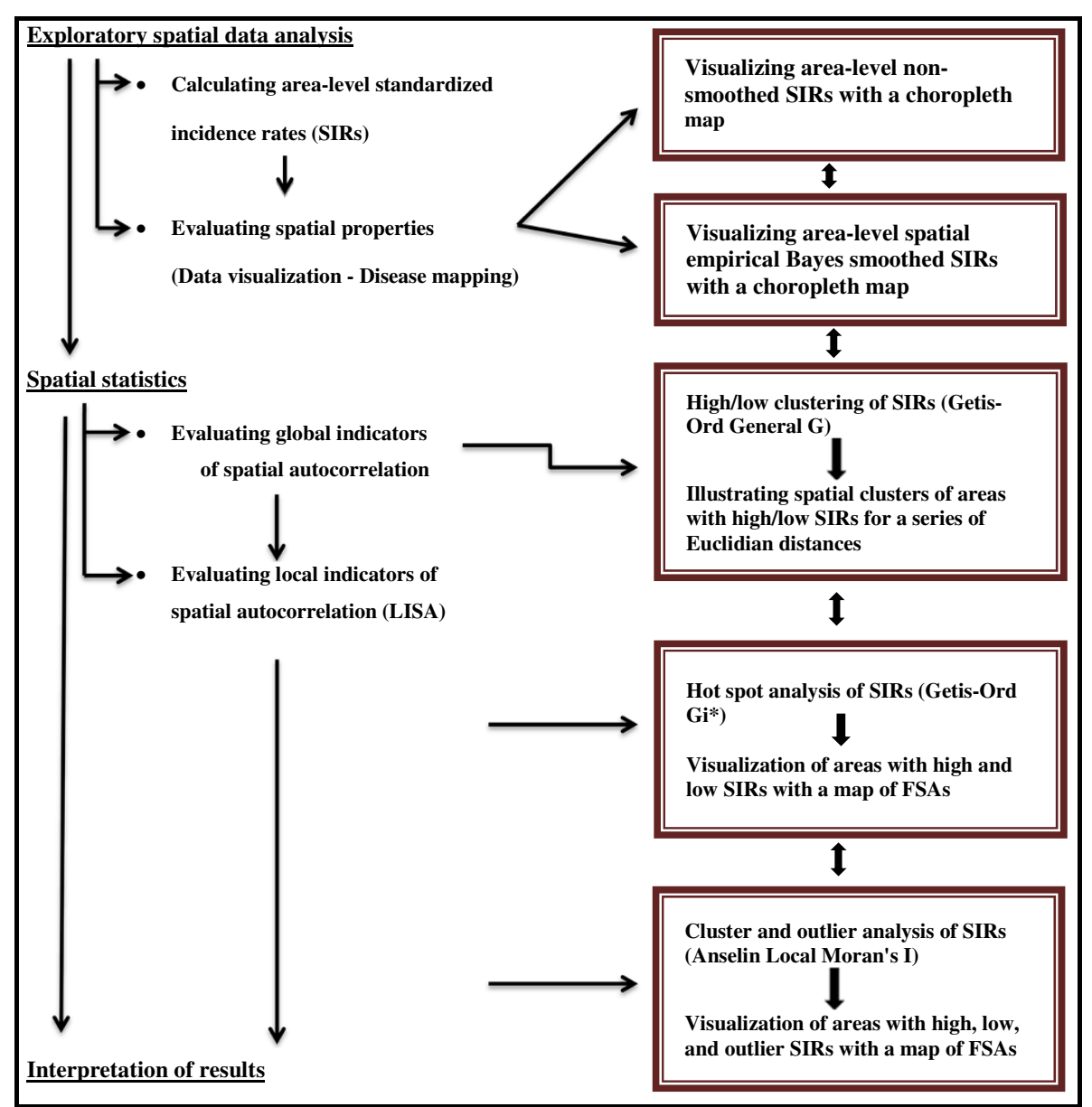

Fig. 2 Flow chart outlining the analytical steps used to evaluate area-level Salmonella Enteritidis infection rates

\section{Exploratory spatial analysis}

In order to obtain stable infection rate estimates, we excluded FSAs with less than 500 residents. Annual standardized incidence rates (SIRs) were calculated for each FSA using direct standardization $[45,46]$ in STATA Intercooled 10.1 statistical software (Stata Corporation, College Station, TX, USA). The annual SIR was estimated by calculating the observed rate for each age-sex category within each FSA, and multiplying it by the age-sex population numbers, which were obtained from the 2006 Census of Canada [35]. Age categories were in five-year increments from 0 to $>85$ years [47]. To account for unstable SIRs of areas with small populations [48], we smoothed the rates using the SEB method [49] with $2^{\text {nd }}$ order queen contiguity weights [50] in GeoDa version 095i software (Spatial Analysis Lab, University of Illinois Urbana-Champaign, IL, USA). The nonsmoothed and smoothed annual SIRs were presented as the number of $S$. Enteritidis infections per 100,000 person-years per FSA, and were visualized using choropleth maps with ArcGIS 10.1 (ESRI Inc., Redlands, CA,
USA) using Jenk's categorization [51] to define the critical intervals for mapping. Jenk's natural breaks classification was developed to identify the ideal arrangement of values (e.g. rates) into different classes, by reducing the variance within classes and maximizing the variance between classes [51].

\section{Spatial statistics}

Each FSA was represented by a polygon, its centroid, and its distinct non-smoothed SIR. The Spatial Statistics Tool in ArcGIS 10.1 was used to identify global and local spatial clusters. Euclidean distance bands were used to measure distances from each FSA's centroid to neighbouring FSAs' centroids (see Global clustering (Getis-Ord General G) subsection). To avoid the omission of local factors by imposing sharp neighbourhood boundaries, the "zone of indifference" conceptualization parameter was chosen for our global and local cluster analyses. Using this parameter, the target FSA and all neighbouring FSAs within a specified distance band are given a maximum weight; once this critical distance is 
exceeded, neighbouring FSAs are assigned smaller and smaller weights as the distance from the target FSA increases [52, 53]. The null hypothesis for both global and local cluster analyses is that there is complete spatial randomness (i.e. FSAs with high or low nonsmoothed SIRs are randomly distributed across the study area). The null hypothesis is rejected when FSAs with high or low SIRs are more spatially clustered than would be expected if the underlying spatial processes were truly random. When the null hypothesis is rejected, a Z-score and a p-value are given for the identified cluster [52, 53].

\section{Global clustering (Getis-Ord General G)}

Global spatial clustering of FSAs with high or low nonsmoothed SIRs across Toronto was evaluated using the Getis-Ord General G statistic [53]. Distance bands that required each FSA to have at least one neighbour were manually selected; for our data, the minimum distance band was $3.3 \mathrm{~km}$. Several Euclidean distances (3.3 to $5.9 \mathrm{~km}$, with $100 \mathrm{~m}$ increments) were selected and included in the model to identify the distance bands with the highest and lowest statistically significant Z-scores. A large, positive Z-score (values $\geq 1.96$ ) and a significant $p$-value $(p \leq 0.05)$ signified that FSAs with high SIRs were clustered in the study area, whereas a large, negative Z-score (values $\leq-1.96$ ) and a significant p-value signified that FSAs with low SIRs were clustered in the study area [53].

\section{Local clustering}

For the local cluster analyses, we used the distance band identified at the global clustering step that showed maximum spatial clustering of FSAs with high nonsmoothed SIRs (see Global clustering (Getis-Ord General G) subsection).

\section{Hot spot analysis (Getis-Ord Gi*)}

Local spatial clusters of FSAs with high or low nonsmoothed SIRs were examined using the Getis-Ord Gi* statistic $[53,54]$. The statistic compares the local sum of SIRs (the sum of the SIR of the targeted FSA and its neighbouring FSAs) to the sum of SIRs of all FSAs within the study area. A statistically significant large, positive Z-score signifies a local high-rate cluster (hot spot). Hot spots are detected when FSAs with high rates are surrounded by FSAs with high rates; the observed local sum of SIRs is higher than the expected local sum and the difference is too large to be the result of chance alone. Similarly, a statistically significant large, negative Z-score signifies a local low-rate cluster (cold spot), where FSAs with low rates are surrounded by FSAs with low rates [51-54]. Statistically significant hot and cold spots were visualized using a map with FSA boundaries.

\section{Cluster and outlier analysis (Anselin Local Moran's I)}

We also used the Local Moran's I statistic to identify local spatial clusters of FSA-level non-smoothed $S$. Enteritidis SIRs during the study period [55]. The statistic identifies hot spots (high-high), cold spots (low-low), and spatial outliers (high-low and low-high). A positive Local Moran's I value indicates that the target FSA is surrounded by FSAs with similar rates (high-high: FSA with a high rate surrounded by FSAs with high rates; low-low: FSA with a low rate surrounded by FSAs with low rates). A negative Local Moran's I value indicates that the target FSA is surrounded by FSAs with dissimilar rates (high-low: FSA with a high rate surrounded by FSAs with low rates; low-high: FSA with a low rate surrounded by FSAs with high rates) [55]. The designation of FSAs to these four classes depends on the results of a statistical test. This test performs random comparisons among the target FSA's and its neighbours Moran's I values to all FSAs' Moran's I values within the study area, and compares the observed Moran's I value to the value corresponding to the random permutations (expected Moran's I value) [55]. If the test is significant $(p \leq 0.05)$, the observed Moran's I value is significantly larger (or smaller in the case of a negative relationship) than the expected Moran's I value. If the test is not significant, the FSA remains in a neutral class (no spatial dependence) [55]. Statistically significant highhigh, low-low, and outlier local clusters were visualized using a map with FSA boundaries. The two local cluster analytical methods were compared to evaluate their efficacy in identifying local infection clusters (e.g. sensitivity analysis).

\section{Ethics review}

The University of Guelph Ethics Review Board was consulted since we used surveillance data for a reportable disease of humans; however, ethics approval was not required because our data did not contain any personal or health information that could be connected back to the original identifiers.

\section{Results}

\section{Descriptive statistics}

Based on the 2006 Census, there were a total of 102 FSAs in the city of Toronto; the FSA-level population size ranged from 5 to 65,125 persons. Ninety-five FSAs met the inclusion criteria, for which the population size ranged from 2,165 to 65,125 persons (mean $=26,345$ ). A total of 495 laboratory confirmed $S$. Enteritidis infections were identified in the MOHLTC's iPHIS database during the study period (165 cases in 2007, 168 in 2008, and 162 in 2009). In total, 22 cases (4.4\%) were excluded because of missing FSA data (14 cases in 2007, 4 in 2008, and 4 in 2009). Thus, there were 473 


\section{A) Non-smoothed standardized incidence rates (SIR) of Salmonella Enteritidis} infections

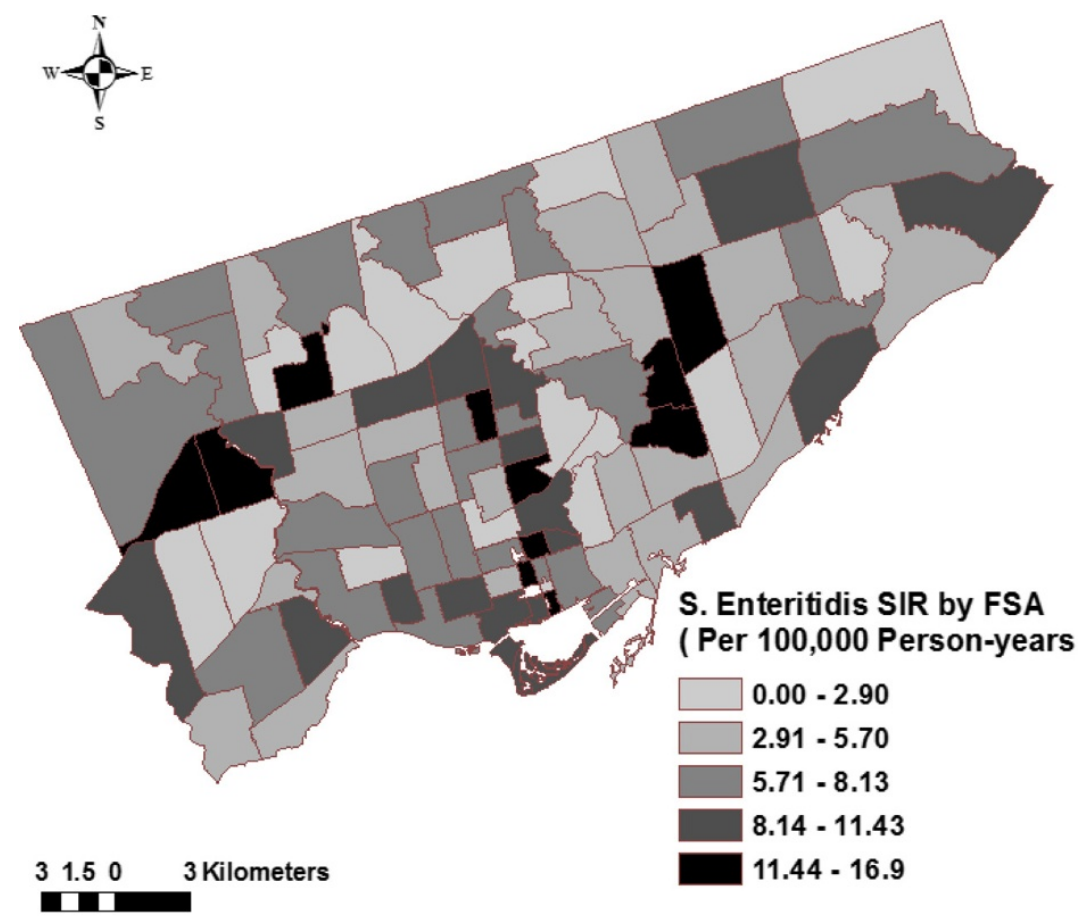

B) Spatial Empirical Bayes smoothed SIR of Salmonella Enteritidis infections

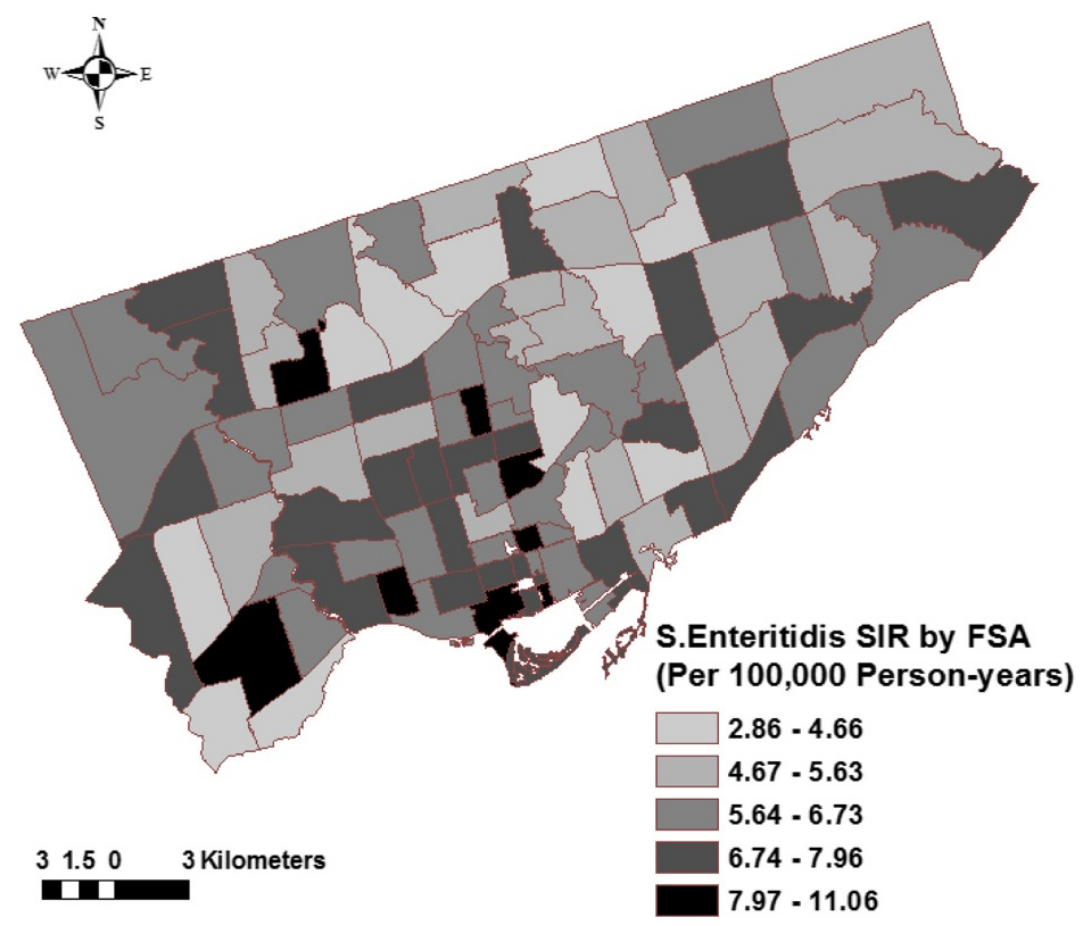

Fig. 3 Distribution of non-smoothed (A) and smoothed (B) Salmonella Enteritidis infection rates in Toronto, $2007-2009$ ( $n=473$ cases; $n=95$ forward sortation areas). Direct standardization was used to calculate forward sortation area (FSA)-level annual standardized incidence rates (SIRs) of Salmonella Enteritidis infections. Spatial empirical Bayes smoothing method with 2nd order queen contiguity weights in GeoDa software (Spatial Analysis Lab, University of Illinois Urbana-Champaign, IL, USA) was used to smooth the SIRs. Maps prepared in ArcGIS 10.1 (ESRI Inc., Redlands, CA, USA) 
cases (151 in 2007, 164 in 2008, and 158 in 2009) available for analysis. No outbreaks were declared by the MOHLTC during the study period.

\section{Exploratory spatial analysis}

Non-smoothed and smoothed standardized incidence rates

Figure 3 illustrates the non-smoothed and smoothed annual SIRs of $S$. Enteritidis infections per FSA in Toronto. The non-smoothed FSA-level SIRs ranged from 0 to 16.9 infections per 100,000 person-years $($ mean $=6.6)$. The smoothed SIRs ranged from 2.9 to $11.1($ mean $=6.3)$.

\section{Spatial statistics}

\section{Global clustering (Getis-Ord General G)}

The Getis-Ord General G statistic results are shown in Figs. 4 and 5. Statistically significant positive Z-scores (1.99 - 2.34) were observed between 3.3 and $4.7 \mathrm{~km}$. The highest statistically significant positive Z-score was observed at $3.3 \mathrm{~km}(\mathrm{Z}=2.34, p=0.019)$, signifying maximum spatial clustering of FSAs with high SIRs at this distance band (Fig. 5). There were no statistically significant negative Z-scores.

\section{Local clustering}

Hot spot analysis (Getis-Ord Gi*)

Eight FSAs with high SIRs (hot spots) (M5C, M5E, M5G, M5M, M5R, M5S, M5T, M9R) and one FSA with a low SIR (cold spot) (M3H) were detected using the

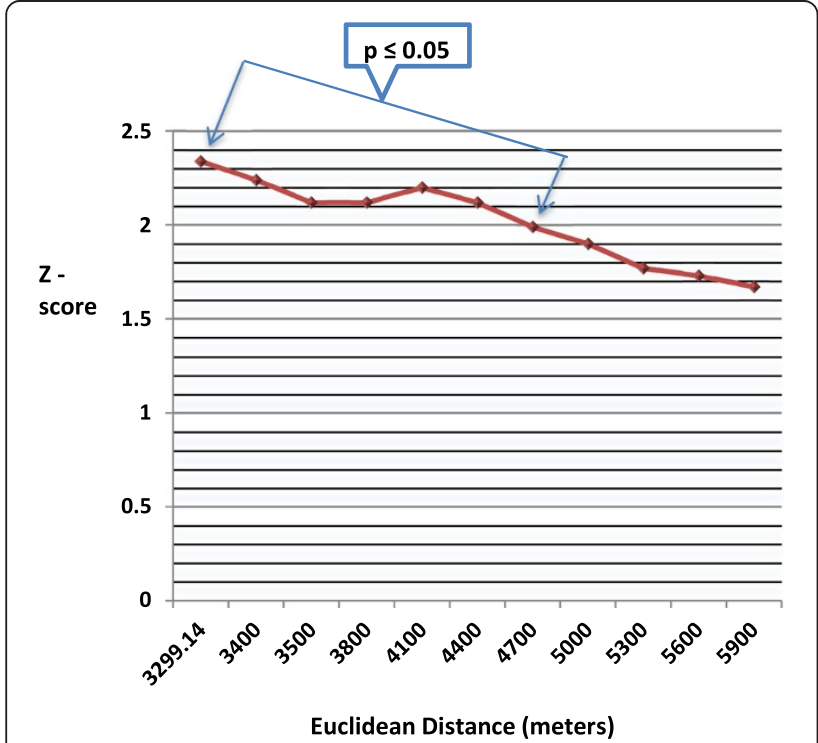

Fig. 4 Global clusters of areas with high Salmonella Enteritidis infection rates in Toronto at different distances. Results of the Getis-Ord G statistic. Large, positive Z-scores (e.g. values $\geq 1.96$ ) indicate global clustering of forward sortation areas with high standardized incidence rates. The zone of indifference conceptualization parameter was used for the analysis. Statistically significant at $p \leq 0.05$
Getis-Ord Gi* method (Table 1, Fig. 6, Additional file 1: Legend 1). The majority of hot spots (6 of 8) were located in south-central (i.e. downtown) Toronto.

\section{Cluster and outlier analysis (Anselin Local Moran's I)}

Five FSAs with high-high SIRs (M4Y, M5E, M5G, M5M, M9R), one FSA with a low-low SIR (M3H), and four outlier FSAs (one high-low (M3M) and three lowhigh (M4G, M5C, M5R)) were identified using the Local Moran's I method (Table 1, Fig. 7, Additional file 1: Legend 1). Three FSAs with high-high SIRs were detected in downtown Toronto.

\section{Discussion}

Salmonella Enteritidis infection rates clustered globally and locally in the city of Toronto. The small distance band at which high $S$. Enteritidis infection rates clustered globally suggests that infection rates were localized to small distinct areas. This finding was subsequently supported by the local cluster analyses, where distinct FSAs, mainly in downtown Toronto, were identified as areas with significantly high SIRs. The two local cluster detection methods (Getis-Ord Gi* and Local Moran's I) identified a number of the same clusters, suggesting consistency between these methods, and indicating the robustness of our study results.

We assessed the area-level spatial heterogeneity of $S$. Enteritidis infection rates across the city of Toronto by combining spatial exploratory and spatial statistical methods with GIS. A systematic approach was used, in which analytical steps succeeded each other, starting from more general to more specific stages that increased our study's specificity. Each step provided additional information to enhance our understanding of the spatial epidemiology of $S$. Enteritidis infection rates in Toronto. However, these steps were sometimes connected and difficult to delineate; consequently, a holistic approach that considers the results of all steps should be followed when interpreting our findings.

The variability of small scale infection rate estimates was accounted for by using the SEB smoothing method. This method reduces the variation of infection rate estimates of areas with unbalanced rates, by shrinking the less stable estimates toward the local mean if local clustering of high-rate areas are detected, and toward the global mean if no local clustering is present [48]. The major advantage of smoothing is that it focuses attention on the overall spatial disease trends, which increases the ability to identify areas with high or low rates. However, as noted with our data, areas can be misclassified by the smoothing method. For example, one high-rate area (M9R) that was evident on the nonsmoothed SIR map and subsequently detected by both local cluster detection methods, was hidden by the 


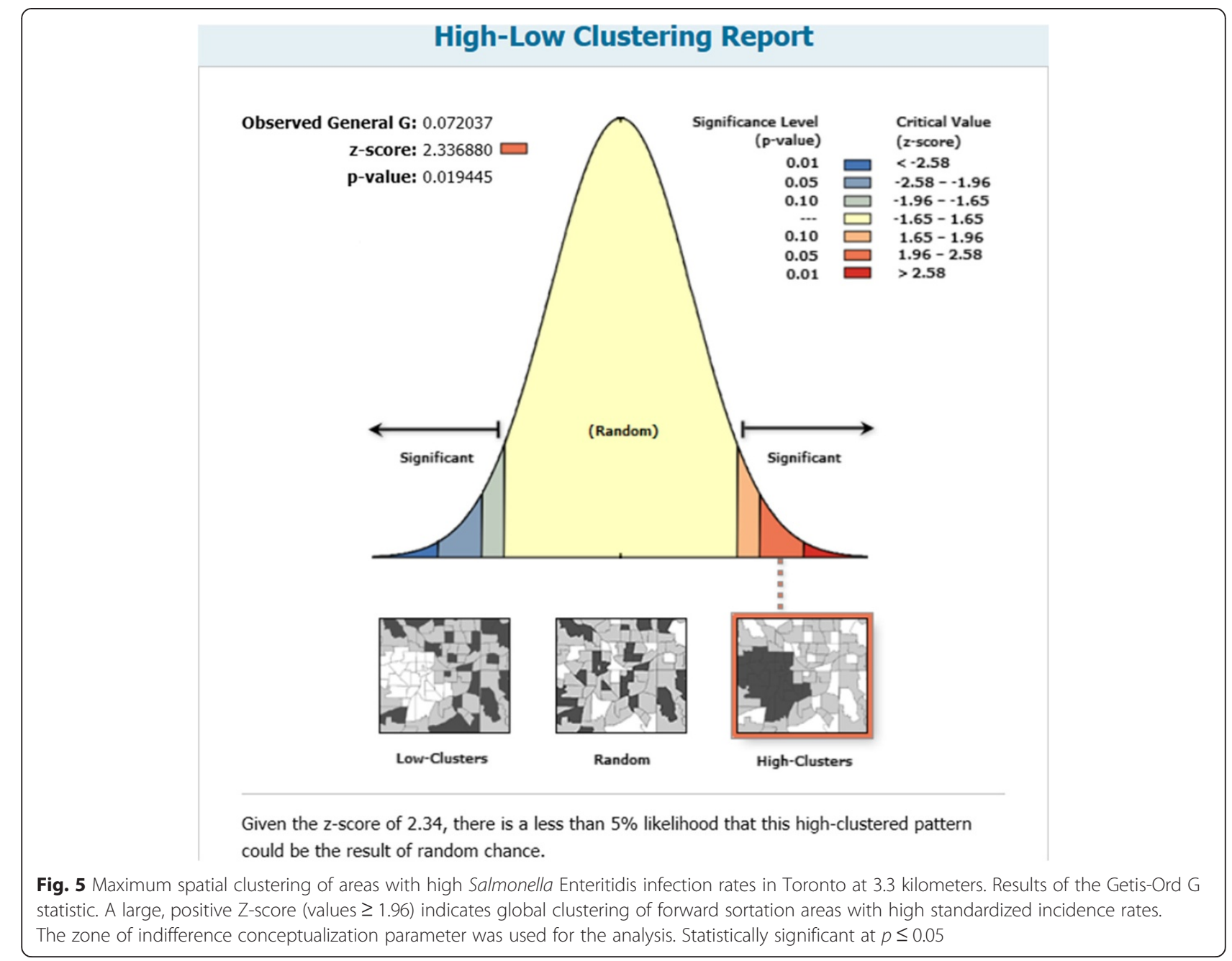

smoothing process. Fewer FSAs with high rates were identified using the smoothed SIRs compared to the non-smoothed SIRs; nonetheless, in the central and south-central parts of the city, both methods identified many of the same high-rate FSAs. The SEB smoothing method reduced the highest non-smoothed SIR by 5.8 units, indicating that there were FSAs with unstable SIR estimates.
When analyzing small scale area-level data, the spatial estimates can become unbalanced at the study area limits where FSAs do not have neighbours. Moreover, because FSAs' boundaries are arbitrary delimitations based on the mail distribution zones of postal facilities, they might not always delineate areas based on their spatial characteristics. To account for potential "edge" and "zoning" effects, we used the "zone of

Table 1 Forward sortation areas identified by different local cluster detection methods

\begin{tabular}{lll}
\hline Type of cluster & Method & Forward sortation area \\
\hline High (hot spot) & Getis-Ord Gi* & M5C, M5E, M5G, M5M, M5R, M5S, M5T, M9R \\
Low (cold spot) & Getis-Ord Gi* & M3H \\
High-high & Local Moran's I & M4Y, M5E, M5G, M5M, M9R \\
Low-low & Local Moran's I & M3H \\
High-low & Local Moran's I & M3M \\
Low-high & Local Moran's I & M4G, M5C, M5R \\
\hline
\end{tabular}




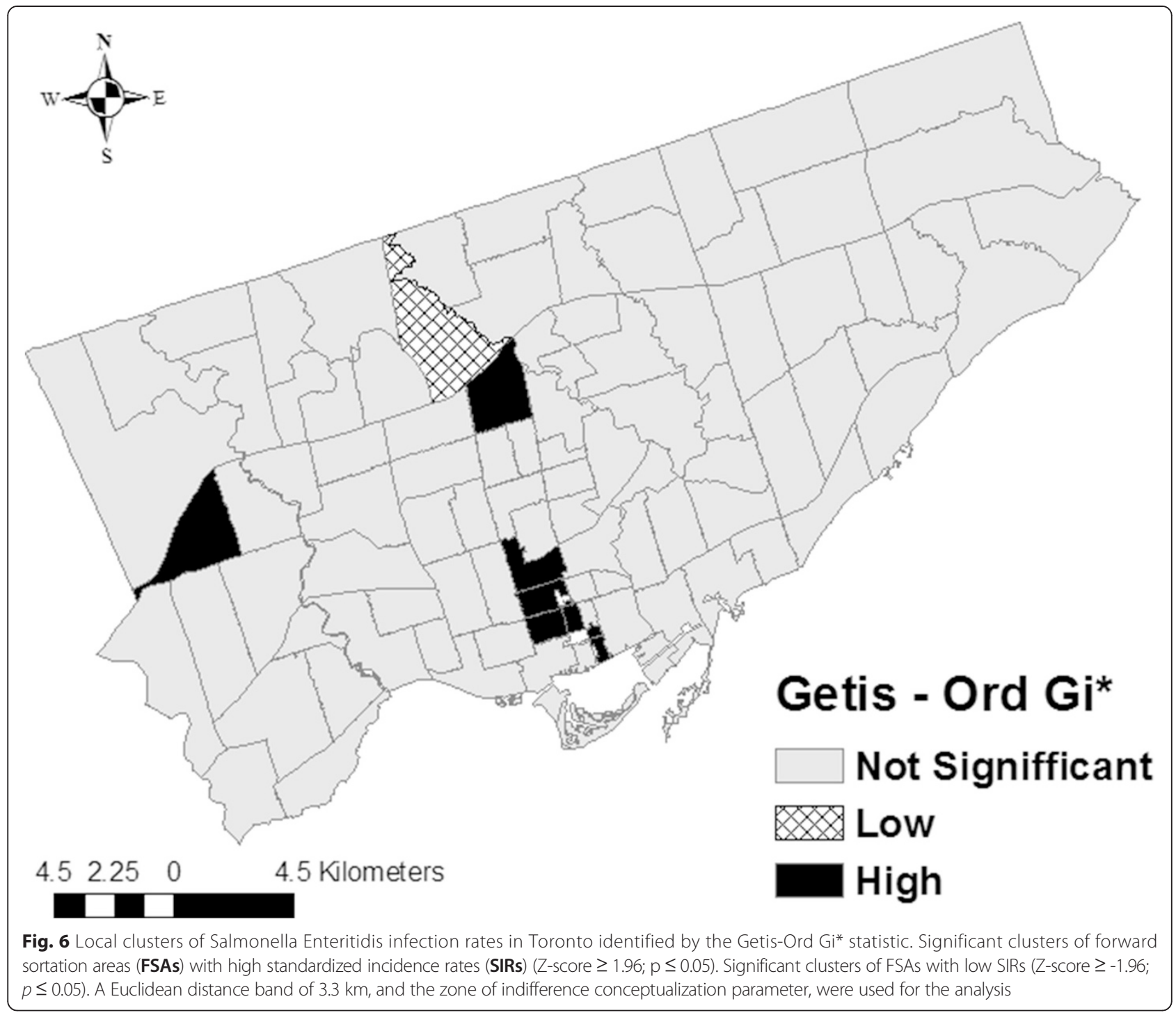

indifference" conceptualization parameter, which does not force sharp boundaries on neighbouring FSA's spatial characteristics nor limit the number of neighbours [52]. This conceptualization parameter considers every FSA to be a neighbour of every other FSA, yet it assigns a maximum weight to areas within a predetermined distance band, and reduces the intensity of spatial relationships once this distance is passed.

The Getis-Ord G method was valuable for identifying the extent of global clustering. Although Toronto is a large city (area of approximately $630 \mathrm{~km}^{2}$ ), maximum spatial clustering of FSAs with high SIRs was detected at $3.3 \mathrm{~km}$, which suggests that clustering of $S$. Enteritidis infections was localized to relatively small areas within the city. This result might suggest that local clusters were driven by small outbreaks (e.g. exposures in homes, local daycares, or restaurants) and not by widespread contamination of food or water supplies. Although outbreak cases are reported to iPHIS and investigated, no local outbreaks were declared by the local public health authorities during the study period.

The Getis-Ord Gi* and Local Moran's I methods identified several of the same clusters. Specifically, four hot spots (M5E, M5G, M5M, M9R) and one cold spot $(\mathrm{M} 3 \mathrm{H})$ were identified by both methods, highlighting the robustness of our study findings. Moreover, our study results are generally in agreement with our previous study [15], in which we evaluated area-level spatial clustering of $S$. Enteritidis infection rates within three public health units (the City of Toronto, Peel Region, and York Region) in the Greater Toronto Area using a spatial discrete Poisson model within a spatial scan statistic. In that study, a single cluster of significantly higher than expected infection rates located in the 


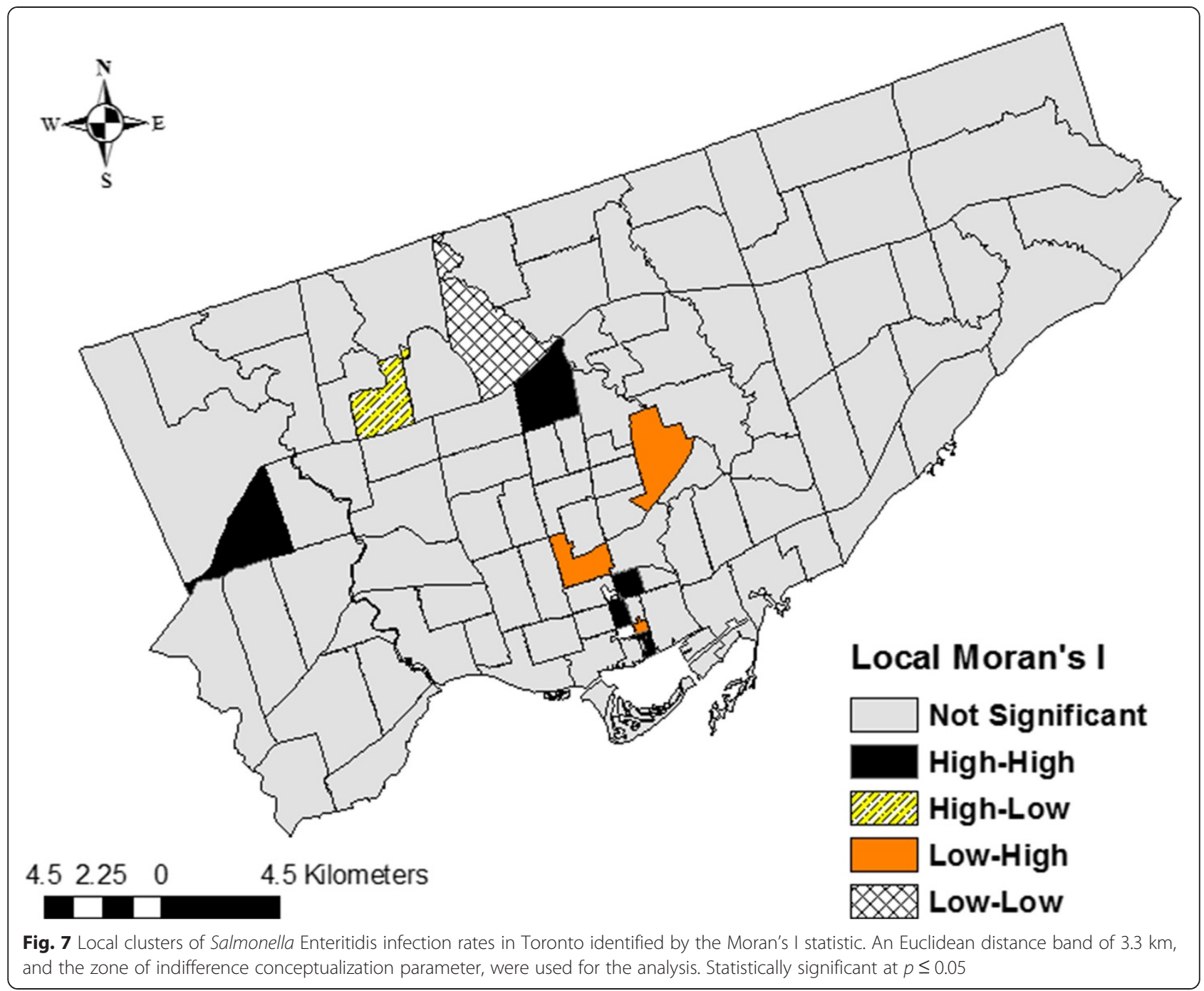

south-central part (downtown) of the City of Toronto Health Unit was identified, which included nine neighbouring FSAs (M4Y, M5B, M5C, M5E, M5G, M5S, M5T, M5V, M6J). By comparison, in the current study, the Getis-Ord $\mathrm{Gi}^{*}$ method detected five hot spots (M5C, M5E, M5G, M5S, M5T), and the Local Moran's I method detected three high-high clusters (M4Y, M5E, M5G) and one low-high cluster (M5C) in downtown Toronto. Taken together, these findings show that these spatial methods could be used in real-time for foodborne disease surveillance data analysis or retrospectively for prevention and control program planning.

However, it is important to understand the specifics of each method to avoid making misleading conclusions. The Getis-Ord Gi* method is ideal when there is an assumption that infection rates cluster within the study area, when investigators are only interested in detecting local high- or low-rate clusters, and when there are a limited number of neighbouring areas with dissimilar rates. Because the Getis-Ord $\mathrm{Gi}^{*}$ statistic includes the target FSA's rate when calculating the local sum of rates, it is not as useful in study areas in which there are several small areas with dissimilar rates. For example, if the target FSA has a sufficiently high rate, it can be designated as a hot spot even though it is surrounded by FSAs with low rates. Likewise, some of its neighbouring low-rate FSAs will also be identified as hot spots; or high- or low-rate FSAs will not be identified at all. These issues explain why two of the hot spots (M5C, M5R) identified by the Getis-Ord Gi* method were identified as low-high clusters by the Local Moran's I method, and why an FSA with a high SIR (M3M) and an FSA with a low SIR (M4G) were undetected by the Getis-Ord Gi* method yet were identified as a high-low and a low-high cluster, respectively, by the Local Moran's I method. The latter method 
identifies local areas with dissimilar rates and excludes these from the local high- or low-rate clusters, thus preventing misclassification of FSAs in study areas with relatively high numbers of dissimilar neighbouring areas.

This study was a hypothesis-generating study and did not aim to identify individual-level risk factors that might influence the spatial heterogeneity of $S$. Enteritidis infection rates. However, both demographic and socioeconomic characteristics have been identified as important risk factors for salmonellosis, some of which include eating behaviours (e.g. frequency of eating outside the home) [6], international travel patterns of local residents $[10,11]$, ethnicity (e.g. proportion of the population that is non-Caucasian) [12], and the proportion of the population with a high income $[12,13,15]$. Moreover, local clusters of high $S$. Enteritidis infection rates could be explained by differences in environmental contamination of food products in local retail facilities and restaurants [56], variations in microbial quality of food consumed [57], or food safety practices followed by local residents [58]. Future hypothesis-testing studies should be conducted in high-rate FSAs to identify area- and individuallevel environmental, behavioural, and socioeconomic risk factors that impact $S$. Enteritidis infection rates. Areas identified as spatial outliers should be investigated using case-control studies (e.g. high-rate areas designated as cases and low-rate areas designated as controls) to identify risk factors that contribute to infection rate increases.

As with every population-based ecological study, our research has limitations, which should be considered when interpreting our results. We recognize that analysis at a different scale might offer different results (the "modifiable areal unit problem") $[19,59]$. However, previous studies have demonstrated that examining infection rates at small scales reduces ecological bias, and gives optimal estimates for area-level risk factors for foodborne diseases [19, 60, 61]. The "zoning effect" [62] might also have occurred if neighbourhood boundaries did not follow the area's spatial characteristics. However, Toronto's FSAs are of a sufficiently small scale to highlight and delimit neighbourhoods with distinct spatial characteristics, and we also accounted for this issue by using the "zone of indifference" conceptualization parameter. Another limitation of our study is that passive surveillance systems underdiagnose and underreport the true level of infection [2, 63, 64]. Population changes might also have occurred during the study period due to movement of residents into and out of the study area. However, this issue should be minor because populations generally do not change considerably in a relatively short time frame. Lastly, exclusion of cases due to missing information might have affected our results. However, $96 \%$ of available cases were included in our analysis; therefore, our estimates should be reliable.

\section{Conclusions}

To the best of our knowledge, this is the first study worldwide that investigated the spatial epidemiology of $S$. Enteritidis infections in an urban setting. Salmonella Enteritidis infection rates clustered globally at a small distance band of $3.3 \mathrm{~km}$, suggesting clustering of high rates in small distinct areas. This finding was supported by the local cluster analyses, where distinct FSAs with high rates, mainly in downtown Toronto, were detected. The robustness of our research findings were demonstrated by linking a number of spatial data explorations and statistical methods with GIS. Our study findings will aid public health professionals to target hypothesisgenerating and hypothesis-testing studies in areas with high $S$. Enteritidis infection rates to generate data for public health interventions.

\section{Additional file}

Additional file 1: Legend for Table 1 and Figs. 6 and 7. Toronto forward sortation area labels. (TIFF $125 \mathrm{~kb}$ )

\section{Abbreviations}

FSA: Forward sortation area; GIS: Geographic information system;

iPHIS: integrated Public Health Information System; MOHLTC: Ontario Ministry of Health and Long-Term Care; SEB: Spatial empirical Bayes; S. Enteritidis: Salmonella enterica serovar Enteritidis; SIR: Standardized incidence rate.

\section{Competing interests}

The authors declare that they have no competing interests.

\section{Authors' contributions}

CV developed the study design, analyzed the data, created the maps, interpreted the results, wrote the first draft of the manuscript, responded to editorial comments, and prepared the final manuscript for submission. MTG and DLP were consulted on study design, data analysis, interpretation of results, and reviewed and commented on manuscript drafts. SAM, FP, and JMS provided advice on the data analysis, interpretation of results, and reviewed and commented on manuscript drafts. All authors read and approved the final manuscript.

\section{Acknowledgements}

The authors acknowledge the MOHLTC for providing the data. We thank the staff of all public health units and public health laboratories that tested samples, followed up with cases, and entered information into the public health surveillance database. We also acknowledge the Data Resource Centre at the University of Guelph library for their GIS and census support. The views expressed in this study are the views of the authors and do not necessarily reflect those of the MOHLTC.

\section{Author details}

'Department of Population Medicine, Ontario Veterinary College, University of Guelph, Guelph, ON N1G 2W1, Canada. ${ }^{2}$ Ontario Ministry of Agriculture, Food and Rural Affairs, Guelph, ON N1G 4Y2, Canada. ${ }^{3}$ Centre for Public Health and Zoonoses, Ontario Veterinary College, University of Guelph, Guelph, ON N1G 2W1, Canada. ${ }^{4}$ Centre for Foodborne, Environmental and Zoonotic Infectious Diseases, Public Health Agency of Canada, Guelph, ON $\mathrm{N} 1 \mathrm{H}$ 8J1, Canada.

Received: 5 August 2014 Accepted: 14 August 2015

Published online: 21 August 2015 


\section{References}

1. Majowicz SE, Musto J, Scallan E, Angulo FJ, Kirk M, O'Brien SJ, et al. International Collaboration on Enteric Disease 'Burden of Illness' Studies: The global burden of nontyphoidal Salmonella gastroenteritis. Clin Infect Dis. 2010;50(6):882-9.

2. Thomas MK, Murray R, Flockhart L, Pintar K, Pollari F, Fazil A, et al. Estimates of the Burden of Foodborne IIIness in Canada for 30 Specified Pathogens and Unspecified Agents, Circa 2006. Foodborne Pathog Dis. 2013;10(7):639-48

3. Nesbitt A, Ravel A, Murray R, McCormick R, Savelli C, Finley R, et al. Integrated surveillance and potential sources of Salmonella Enteritidis in human cases in Canada from 2003 to 2009. Epidemiol Infect. 2012;140(10):1757-72.

4. Chai SJ, White PL, Lathrop SL, Solghan SM, Medus C, McGlinchey BM, et al. Salmonella enterica serotype Enteritidis: increasing incidence of domestically acquired infections. Clin Infect Dis. 2012;54 Suppl 5:S488-97.

5. European Food Safety Authority (EFSA). The Community Summary Report on Trends and Sources of Zoonoses, Zoonotic Agents and food-borne outbreaks in the European Union in 2008. EFSA J. 2010;8(1):1496.

6. Kimura AC, Reddy V, Marcus R, Cieslak PR, Mohle-Boetani JC, Kassenborg $H D$, et al. Chicken consumption is a newly identified risk factor for sporadic Salmonella enterica serotype Enteritidis infections in the United States: a case-control study in FoodNet sites. Clin Infect Dis. 2004;38 Suppl 3:S244-52.

7. Marcus R, Varma JK, Medus C, Boothe EJ, Anderson BJ, Crume T, et al. Reassessment of risk factors for sporadic Salmonella serotype Enteritidis infections: a case-control study in five Food Net Sites, 2002-2003. Epidemiol Infect. 2007;135(1):84-92.

8. Taylor M, Leslie M, Ritson M, Stone J, Cox W, Hoang L, et al. Investigation of the Concurrent Emergence of Salmonella Enteritidis in Humans and Poultry in British Columbia, Canada, 2008-2010. Zoonoses Public Health. 2012;59(8):584-92.

9. Braden CR. Salmonella enterica serotype Enteritidis and eggs: a national epidemic in the United States. Clin Infect Dis. 2006;43(4):512-7.

10. Johnson LR, Gould LH, Dunn JR, Berkelman R, Mahon For The Foodnet Travel Working Group BE. Salmonella Infections Associated with International Travel: A Foodborne Diseases Active Surveillance Network (FoodNet) Study. Foodborne Pathog Dis. 2011;8(9):1031-7.

11. Tighe MK, Savage R, Vrbova L, Toolan M, Whitfield Y, Varga C, et al. The epidemiology of travel-related Salmonella Enteritidis in Ontario, Canada, 2010-2011. BMC Public Health. 2012;12(1):310.

12. Chang M, Groseclose SL, Zaidi AA, Braden CR. An ecological analysis of sociodemographic factors associated with the incidence of salmonellosis, shigellosis, and E. coli O157:H7 infections in US counties. Epidemiol Infect. 2009;137(6):810-20.

13. Varga C, Pearl DL, McEwen SA, Sargeant JM, Pollari F, Guerin MT. Incidence, distribution, seasonality, and demographic risk factors of Salmonella Enteritidis human infections in Ontario, Canada, 2007-2009. BMC Infect Dis. 2013;13:212.

14. Younus M, Hartwick E, Siddiqi AA, Wilkins M, Davies HD, Rahbar M, et al. The role of neighborhood level socioeconomic characteristics in Salmonella infections in Michigan (1997-2007): assessment using geographic information system. Int J Health Geogr. 2007;6:56.

15. Varga C, Pearl DL, McEwen SA, Sargeant JM, Pollari F, Guerin MT. Evaluating area-level spatial clustering of Salmonella Enteritidis infections and their socioeconomic determinants in the Greater Toronto Area, Ontario, Canada (2007 - 2009): A retrospective population-based ecological study. BMC Public Health. 2013;13:1078.

16. Younus M, Wilkins MJ, Davies HD, Rahbar MH, Funk J, Nguyen C, et al. The role of exposures to animals and other risk factors in sporadic, non-typhoidal Salmonella infections in Michigan children. Zoonoses Public Health. 2010;57(7-8):170-6.

17. Pardhan-Ali A, Berke O, Wilson J, Edge VL, Furgal C, Reid-Smith R, et al. A spatial and temporal analysis of notifiable gastrointestinal illness in the Northwest Territories, Canada, 1991-2008. Int J Health Geogr. 2012;11:17.

18. Odoi A, Martin SW, Michel P, Middleton D, Holt J, Wilson J. Investigation of clusters of giardiasis using GIS and a spatial scan statistic. Int J Health Geogr. 2004;3(1):11.

19. Arsenault J, Berke O, Michel P, Ravel A, Gosselin P. Environmental and demographic risk factors for campylobacteriosis: Do various geographical scales tell the same story? BMC Infect Dis. 2012;12(1):318.

20. Green CG, Krause DO, Wylie JL. Spatial analysis of campylobacter infection in the Canadian province of Manitoba. Int J Health Geogr. 2006;5:2.

21. Stark JH, Sharma R, Ostroff S, Cummings DA, Ermentrout B, Stebbins S, et al. Local spatial and temporal processes of influenza in Pennsylvania, USA: 2003-2009. PLoS ONE. 2012;7(3):e34245.
22. Pearl DL, Louie M, Chui L, Doré K, Grimsrud KM, Leedell D, et al. The use of outbreak information in the interpretation of clustering of reported cases of Escherichia coli 0157 in space and time in Alberta, Canada, 2000-2002. Epidemiol Infect. 2006;134(4):699-711.

23. So HC, Pearl DL, von Königslöw T, Louie M, Chui L, Svenson LW. Spatiotemporal scan statistics for the detection of outbreaks involving common molecular subtypes: using human cases of Escherichia coli 0157:H7 provincial PFGE pattern 8 (National Designation ECXAl.0001) in Alberta as an example. Zoonoses Public Health. 2013;60(5):341-8.

24. Ali M, Wagatsuma Y, Emch M, Breiman RF. Use of a geographic information system for defining spatial risk for dengue transmission in Bangladesh: role for Aedes albopictus in an urban outbreak. Am J Trop Med Hyg. 2003;69(6):634-40

25. Alzahrani AG, Al Mazroa MA, Alrabeah AM, Ibrahim AM, Mokdad AH, Memish ZA. Geographical distribution and spatio-temporal patterns of dengue cases in Jeddah Governorate from 2006-2008. Trans R Soc Trop Med Hyg. 2013;107(1):23-9.

26. Colantonio A, Moldofsky B, Escobar M, Vernich L, Chipman M, McLellan B. Using geographical information systems mapping to identify areas presenting high risk for traumatic brain injury. Emerg Themes Epidemiol. 2011;8:7.

27. Pedigo A, Aldrich T, Odoi A. Neighborhood disparities in stroke and myocardial infarction mortality: a GIS and spatial scan statistics approach. BMC Public Health. 2011;11:644.

28. Wilking $H$, Höhle M, Velasco E, Suckau M, Tim E. Ecological analysis of social risk factors for Rotavirus infections in Berlin, Germany, 2007-2009. Int J Health Geogr. 2012;11(1):37.

29. Lee SS, Wong NS. The clustering and transmission dynamics of pandemic influenza A (H1N1) 2009 cases in Hong Kong. J Infect. 2011;63(4):274-80.

30. Wang T, Xue F, Chen Y, Ma Y, Liu Y. The spatial epidemiology of tuberculosis in Linyi City, China, 2005-2010. BMC Public Health. 2012;12:885.

31. Corner RJ, Dewan AM, Hashizume M. Modelling typhoid risk in Dhaka Metropolitan Area of Bangladesh: the role of socio-economic and environmental factors. Int J Health Geogr. 2013;12(1):13.

32. Dewan AM, Corner RJ, Hashizume M. Modelling spatiotemporal patterns of typhoid cases between 2005 and 2009 using spatial statistics. In: Dhaka Megacity. Netherlands: Springer; 2014. p. 345-65.

33. Dewan AM, Corner R, Hashizume M, Ongee ET. Typhoid fever and its association with environmental factors in the Dhaka metropolitan area of Bangladesh: a spatial and time-series approach. PLoS Negl Trop Dis. 2013;7(1):e1998.

34. Ali S, Corner RJ, Hashizume M. Spatiotemporal analysis of dengue infection between 2005 and 2010. In: Dewan A, Corner R, editors. Dhaka Megacity. Geospatial Perspectives on Urbanisation, Environment and Health. Netherlands: Springer; 2014. p. 367-84.

35. Statistics Canada: Population and dwelling counts, for Canada, provinces and territories, and forward sortation areas as reported by the respondents, 2006 Census. Available at: http://www12.statcan.ca/english/census06/data/ popdwell/Table.cfm? $T=1201 \& S R=1 \& S=0 \& O=A \& R P P=9999 \& P R=0 \& C M A=0$

36. Huang CF, Chen PL, Liu MF, Lee CC, Lee NY, Chang CM, et al. Nontyphoidal Salmonella bacteremia in patients with connective tissue diseases. $J$ Microbiol Immunol Infect. 2012;45(5):350-5.

37. Zaidenstein R, Peretz C, Nissan I, Reisfeld A, Yaron S, Agmon V, et al. The epidemiology of extraintestinal non-typhoid Salmonella in Israel: the effects of patients' age and sex. Eur J Clin Microbiol Infect Dis. 2010;29(9):1103-9.

38. Chui KK, Webb P, Russell RM, Naumova EN. Geographic variations and temporal trends of Salmonella-associated hospitalization in the U.S. elderly, 1991-2004: a time series analysis of the impact of HACCP regulation. BMC Public Health. 2009;9:447.

39. Lawson AB. Statistical Methods in Spatial Epidemiology. New York: Wiley; 2006.

40. Elliott $P$, Savitz DA. Design issues in small-area studies of environment and health. Environ Health Perspect. 2008;116(8):1098-104.

41. Statistics Canada: 2006 - Census Forward Sortation Area Boundary File, Reference Guide. Available at: http://www.statcan.gc.ca/bsolc/olc-cel/olccel?catno=92-170-GWE\&lang=eng

42. Government of Ontario. Health Protection and Promotion Act. Revised Statutes of Ontario. 1990.

43. Ontario Ministry of Health and Long-Term Care (MOHLTC). Ontario Public Health Standards. Infectious Diseases Protocol. 2013. Available at: http:// www.health.gov.on.ca/en/pro/programs/publichealth/oph_standards/ infdispro.aspx. 
44. Bale JA, de Pinna EM, Threlfall EJ, Ward LR. Kauffmann-White Scheme 2007: Salmonella Identification - Serotypes and Antigenic Formulae. London: Health Protection Agency; 2007.

45. Curtin LR, Klein RJ. Direct Standardization (Age-Adjusted Death Rates). Centres for Disease Control and Prevention: Healthy People 2000. Stat Notes. 1995;6:1-10.

46. Ahmad OB, Boschi-Pinto C, Lopez AD, Murray CJL, Lozano R, Inoue M. Age standardization of rates: a new WHO world standard. (GPE Discussion Paper No. 31. EIP/GPE/EBD). Geneva: World Health Organization; 2000.

47. Keegan VA, Majowicz SE, Pearl DL, Marshall BJ, Sittler N, Knowles L, et al. Epidemiology of enteric disease in C-EnterNet's pilot site - Waterloo region, Ontario, 1990 to 2004. Can J Infect Dis Med Microbiol. 2009;20(3):79-87.

48. Clayton D, Bernardinelli L. Bayesian methods for mapping disease risk. In: Elliott CJ, English D, Stern R, editors. Geographical and Environmental Epidemiology. Oxford: Oxford University Press; 1997. p. 206-20.

49. Waller L, Gotway C. Mapping smoothed rates and probabilities. New Jersey, John Wiley \& Sons, Inc: Applied Statial Statistics for Public Health Data. Hoboken; 2004. p. 86-104.

50. Anselin L, Syabri I, Kho Y. GeoDa: an introduction to spatial data analysis. Geogr Anal. 2006;38(1):5-22.

51. Jenks GF. The Data Model Concept in Statistical Mapping. International Yearbook of Cartography. 1967;7:186-90

52. Mitchell A. The ESRI Guide to GIS Analysis, vol. 2. Redlands, CA, USA: ESRI Press; 2005.

53. Getis A, Ord JK. The Analysis of Spatial Association by Use of Distance Statistics. Geogr Anal. 1992;24:189-206.

54. Ord JK, Getis A. Local spatial autocorrelation statistics: distributional issues and an application. Geogr Anal. 1995:27:286-306.

55. Anselin L. Local indicators of spatial association - LISA. Geogr Anal. 1995;27:93-115

56. Signs RJ, Darcey VL, Carney TA, Evans AA, Quinlan JJ. Retail food safety risks for populations of different races, ethnicities, and income levels. J Food Prot. 2011:74(10):1717-23.

57. Koro ME, Anandan S, Quinlan JJ. Microbial quality of food available to populations of differing socioeconomic status. Am J Prev Med. 2010;38(5):478-81.

58. Patil SR, Cates S, Morales R. Consumer food safety knowledge, practices, and demographic differences: findings from a meta-analysis. J Food Prot 2005;68:1884-94.

59. Wong D. The modifiable areal unit problem (MAUP). In: Fotheringham AS, Rogerson PA, editors. The SAGE handbook of spatial analysis. London: SAGE publications; 2009. p. 105-23.

60. Weisent J, Rohrbach B, Dunn JR, Odoi A. Detection of high risk campylobacteriosis clusters at three geographic levels. Geospat Health. 2011;6(1):65-76.

61. Morgenstern H. Ecologic studies in epidemiology: concepts, principles, and methods. Annu Rev Public Health. 1995;16:61-81.

62. Waller LA, Gotway CA. Applied spatial statistics for public health data. Hoboken, New Jersey: John Wiley \& Sons Inc; 2004.

63. MacDougall L, Majowicz S, Doré K, Flint J, Thomas K, Kovacs S, et al. Under-reporting of infectious gastrointestinal illness in British Columbia, Canada: who is counted in provincial communicable disease statistics? Epidemiol Infect. 2008;136(2):248-56.

64. Scallan E, Jones TF, Cronquist A, Thomas S, Frenzen P, Hoefer D, et al. 9. Foodborne Pathog Dis. 2006;3(4):432-8.

\section{Submit your next manuscript to BioMed Central and take full advantage of:}

- Convenient online submission

- Thorough peer review

- No space constraints or color figure charges

- Immediate publication on acceptance

- Inclusion in PubMed, CAS, Scopus and Google Scholar

- Research which is freely available for redistribution 